\title{
Integrated approach to the management of patients having cicatricial deformity of the uterine cervix
}

\author{
Svetlana N. Buyanova ${ }^{1}$, Natalya A. Shchukina ${ }^{1}$, Ekaterina I. Petrosyann ${ }^{1}$, Nadezhda V. Dub $^{2}$
}

\begin{abstract}
Introduction: Cicatricial deformity of the uterine cervix is a relevant and understudied problem in medicine. It is important to conduct preoperative preparation aimed at creating a favorable background for operative treatment as well as to conduct post-operative treatment for improving and accelerating the reparative processes.

Objective: Improving the results of surgical treatment of cicatricial deformity of the uterine cervix with the help of preoperative preparation and evaluating the efficiency of application of the Flamena gel complex in the post-operative period.

Method: There were 30 patients having cicatricial deformity of the uterine cervix aged 25 to 45 under observation. With all patients, the clinical laboratory and instrumental examination was conducted which included: microscopic examination of the intensity of leukocytosis in the vaginal and cervical secretion, cytological examination, bacteriological inoculation from the cervical canal, the PCR method test for sexually transmitted infections (the STIs), detection of the human papillomavirus, the content of immunoglobulines ( $\lg A, \lg G, \lg M)$ in the venous blood serum and in the mucous secretion of the cervical canal, colposcopy, ultrasonography, and histological examination of the uterine cervix.

Results: After normalizing the biocenosis of the vagina, the Superlimf 25 units immunomodulating agent was prescribed to all patients, as another preoperative preparation stage. Then surgical treatment was performed. In the post-operative period, the Flamena gel complex was prescribed. The combined therapy contributed to improving the results of the surgical treatment.

Conclusion: The algorithm suggested by the authors which includes preoperative preparation, surgical treatment and post-operative care can be used as an integrated approach to the management of patients having cicatricial deformity of the uterine cervix.
\end{abstract}

Keywords: uterine cervix, lacerations of cervix, preoperative preparation, surgical treatment, post-operative recovery

\section{INTRODUCTION}

Among gynecological diseases, pathology of the uterine cervix is currently an extremely important and relevant problem. Precancer processes and the cervical cancer (CC) are being detected, treated and prevented worldwide. According to the authors' data, in Russia, the CC ranks $2^{\text {nd }}$ among malignant neoplasms in women aged under 45 and it ranks $1^{\text {st }}$ in mortality (1-3). In the USA, the mortality caused by the CC is going down owing to the broadly implemented screening $(2,4)$. In the majority of cases, the CC is registered in women who have not undergone screening $(2,5)$. The cause leading to precancer processes and the $\mathrm{CC}$ is the papilloma viral infection (the PVI). According to the data of the World Health Organization, 2.5-3 million cases of the PVI are diagnosed in the world annually. Over 600 million people are infected with the HPV $(6,7)$. According to the data of Wheeler et al. (2006), in 3 years from the point of being infected with the HPV, a II-III degree cervical intraepithelial neoplasia (Cervical Intraepithelial Lesions, CIN) of the uterine cervix develops in every one in 4 women (27\%) (8,9). In the Russian Federation, the PVI is found in $15-34 \%$ of women in the general population and in $44,9 \%$ of patients being examined for the sexually transmitted infections (STIs). However, while cancer of the uterine cervix, precancer processes and the human papillomavirus are in the focus, not enough attention is paid to questions concerning the management of patients having cicatricial deformity of the uterine cervix.

\footnotetext{
State Budget-Funded Institution of Healthcare of Moscow Region "Moscow Regional Research Institute of Obstetrics and Gynecology" of the Ministry of healthcare of Moscow Region, Moscow, Russia.

2 State Budgetary Institution of Health Care Ministry of the Moscow Region «Moscow Regional Research and Clinical Institute named after M.F. Vladimirskii», Moscow, Russia.
}

Correspondence: Svetlana. N. Buyanova

State Budget-Funded Institution of Healthcare of Moscow Region "Moscow Regional Research Institute of Obstetrics and Gynecology" of the Ministry of healthcare of Moscow Region, Moscow, Russia.

E-mail: buyanova-sn@mail.ru

Received: 1 Aug 2019, Accepted: 20 Sep 2019

(C) 2019 by the authors; licensee Modestum Ltd., UK. This article is an open access article distributed under the terms and conditions of the Creative Commons Attribution License (http://creativecommons.org/licenses/by/4.0/). 
Laceration of cervix is a traumatic disruption of continuity of the uterine cervix during delivery or invasive interventions. The rupture heals by way of cicatrisation, the uterine cervix gets deformed with the formation of the cervical canal being disturbed, and it results into an ectropion. The mucous plug cannot be retained in the cervical canal, so it does not perform its barrier function. Subsequently, conditions are formed for the ascending infection and involvement of the uterine cavity. This problem deserves attention because cicatricial deformity of the uterine cervix is "accompanied" by an inflammatory process, and the inflammatory process persisting for a prolonged time leads to background diseases, and then to the precancer ones. According to a number of studies, it has been found that against the background of cicatricial deformity of the uterine cervix, the LSIL and HSIL lesions occur 3 times more frequently than without any anatomical changes of the cervix $(10,11)$. Another important point is that cicatricial deformity of the uterine cervix can be the cause of infertility, isthmic and cervical insufficiency, pregnancy loss and it can lead to intrauterine infection of the fetus during pregnancy, alongside with infectious and inflammatory processes (11).

The only way to prevent all the above complications and restore the normal biocenosis of the uterine cervix is operative treatment. Restoration of the anatomy of the cervix allows eliminating the functional disorders.

As it has been mentioned earlier, cicatricial deformity of the uterine cervix is characterized by an inflammatory process present. Hence surgical treatment always has to be preceded by preoperative preparation aimed at elimination of the inflammatory process and sexually transmitted infections (STIs), because both the inflammatory process and the STIs affect the progress of post-operative period and regeneration processes adversely. In the scientific literature, they have described cervicites repeatedly, with attempting to point out etiological specific features. Meanwhile, the task is to conduct additional laboratory studies (12-16). An important part is also given to the use of immunomodulating agents 10 days before the operative intervention in order to improve the local immunity (17-18). Alongside with preoperative preparation of the uterine cervix, the relevant post-operative care is essential, too, aimed at improvement and reduction of the recovery term.

All this confirms that treatment of cicatricial deformity of the uterine cervix includes not only surgical intervention but also preoperative preparation and post-operative management.

Objective of the research: improving the results of surgical treatment of cicatricial deformity of the uterine cervix with the help of preoperative preparation and evaluating the efficiency of application of the liposomal gel coating with dihydroquercetin on regenerative properties of tissues in the post-operative period.

\section{MATERIALS AND METHODS}

The research was conducted in the operative gynecology department at the State budget-funded institution of healthcare of Moscow Region "Moscow regional research institute of obstetrics and gynecology" (SBIH MR MRRIOG) within January-August, 2019. The research incorporated the clinical and laboratory examination of 30 female patients aged 25 to 45 having cicatricial deformity of the uterine cervix, with operative treatment indicated for them. All the patients who participated in the research were examined by clinical, laboratory and instrumental methods. The duration of dynamic observation amounted to 2 to 4 months. During this time, several check-up examinations were conducted: before surgical treatment - preoperative preparation and in 2 months after surgical treatment.

The clinical examination included history taking (what the progress of pregnancy was, if there was any isthmic and cervical insufficiency and if it was corrected, what the progress of delivery was, if there were any complications during delivery and in the early postpartum period, parity, weight and height of children at birth, the quantity of abortions, lacerations of cervix during delivery, if the ruptures were sutured, what the patient's reproductive plans are), with evaluation of the quality of life (dyspareunia, vaginal discomfort, abundant prolonged discharge from the reproductive tract), and gynecological examination (localizing the ruptures of the uterine cervix).

The laboratory examination included:

- microscopic examination of the intensity of leukocytosis in the vaginal and cervical secretion. The results were assessed as follows: 1 to 20 leukocytes in the field of view are within the norm, 20 to 50 leukocytes in the field of view

- the moderate leukocytosis, over 50 leukocytes in the field of view - the pronounced leukocytosis.

- cytological examination. The results had 3 grades: no specific findings in the cytogram, the inflammation cytogram, the cytogram of a pronounced inflammation.

- bacteriological inoculation from the cervical canal for identification of antibiotic-sensitive causative agents. The criterion of etiological significance of the agents was their diagnostic titer of $\geq 10^{4} \mathrm{CFU} / \mathrm{ml}$. 
- sexually transmitted infections (STIS) by the PCR method. Among the infections, there were the following: Chlamydia trachomatis, Mycoplasma hominis, Mycoplasma genitalium, Ureaplasma urealyticum, Gardenerella vaginalis, Cytomegalovirus, herpes simplex virus (HSV) of types I and II, and Epstein-Barr virus (EBV).

- detection of the human papillomavirus (HPV). The results were evaluated as follows: either the HPV was not found, or the HPV was found and so the quantitative viral load was identified.

- the content of immunoglobulines of various classes ( $\lg A, \lg G, \lg M$ ) in the venous blood serum and in the mucous secretion of the cervical canal before the treatment and in 2 months after that in order to evaluate the local inflammation of the female lower reproductive tract.

The instrumental examination methods included:

- colposcopic examination was conducted both without processing of the mucous membrane of the uterine cervix (plain colposcopy), and with the subsequent application of $3 \%$ solution of the acetic acid and Lugol's solution (extended colposcopy). When assessing the colposcopic status, the uniform International colposcopic terminology/classification approved in Rio de Janeiro in 2011 was used. The following items were assessed: a satisfactory (there is the junction of stratified squamous and cylindrical epithelia) or unsatisfactory (there is no junction of stratified squamous and cylindrical epithelia) colposcopic condition, the transformation area type, the presence of abnormal colposcopic condition (acetowhite epithelium, mosaic, punctation, iodine-negative areas).

- ultrasonography of the uterine cervix with identifying the dimensions (length, anteroposterior dimension, width), the structure (the presence of mass inclusions, localization and dimensions), the presence of linear hyperechogenic inclusions (cicatricial changes) on days $5-7$ of the menstrual cycle before and after the treatment.

- histological examination (detecting the signs of a chronic or acute inflammatory process, or the absence of one).

\section{RESULTS AND DISCUSSION}

For all the female patients included into the research, the detailed obstetrical and gynecological history was taken that was of especial interest. All patients (100\%) had a history of deliveries complicated with lacerations of the uterine cervix, with 9 patients (30\%) having a macrosomic delivery.

13 patients (43.3\%) had a history of induced abortions, 16 patients $(53.3 \%)$ - that of surgical intervention on the uterine cervix in the form of diathermocoagulation, chemical coagulation and cryodestruction. While taking the history, it was found that 11 patients (36.6\%) had lacerations of the uterine cervix sutured in the early postpartum period, with 9 patients (30\%) not knowing if they had had any suturing of the uterine cervix in the early postpartum period or not (they had no discharge summaries on hand), and 10 patients (33.3\%) having had no suturing of the ruptures. In the patients included into the research, the main reasons for visiting the doctor - obstetrician gynecologist - were as follows: discharge from the reproductive tract in 22 patients (73.3\%), dyspareunia in 8 patients (26.6\%), and vaginal discomfort in 13 patients (43.3\%). It was found that 23 patients (76.6\%) noted a history of cervicites, and 12 patients (40\%) had a STI alongside with the cervicitis. In all episodes of the inflammatory diseases and sexually transmitted infections, 15 patients (50\%) had the relevant treatment earlier while 15 patients (50\%) were not treated.

The gynecological examination found that rough cicatricial deformity was observed in 25 patients (83.3\%), mostly in women having the history of 2 and more deliveries. The ruptures were more frequently located either at 3 and 9 o'clock position or at 6 and 12 o'clock position and they reached the lateral vault in 11 cases (36.6\%). In 8 cases (26.6\%), nonrough cicatricial deformity was found with one lateral rupture only which did not reach the vaginal vault. Mainly, this was registered in the patients having the history of only one delivery.

For all patients having cicatricial deformity of the uterine cervix, microscopic examination of the intensity of leukocytosis in the vaginal and cervical secretion was conducted. During the examination it was found that 8 patients (26.6\%) had leukocytes within the norm, 19 patients (63.3\%) had a moderate leukocytosis, with $3(10 \%)$ having a pronounced leukocytosis.

Cytological screening returned inflammation cytograms in 19 cases (63.3\%), with 9 cases (30\%) having no specific findings in the cytogram and 2 cases (6.6\%) having a pronounced inflammation cytogram.

Detection of the STIs by the PCR method and bacteriological inoculation from the cervical canal for identifying the antibiotic-sensitive causative agents has shown that during screening the mixed infection was found in 22 cases (73.3\%) - a combination of Mycoplasma hominis or Mycoplasma genitalium, Ureaplasma urealyticum, Gardenerella vaginalis. Bacterial vaginosis (Gardenerella vaginalis) was found in 5 patients (16.6\%) as the mono-infection and in 6 cases (20\%) as a mixed one which was mentioned above. In 13 patients (43.3\%), Cytomegalovirus, herpes simplex virus (HSV) of types I and II, and Epstein-Barr virus (EBV) were found both as the mono- and a mixed infection. The Candida genus fungi were 
detected in 14 patients (46.6\%) at the titer of over $\geq 10^{4} \mathrm{CFU} / \mathrm{ml}$, in 4 patients (13.3\%) at less than $10^{4} \mathrm{CFU} / \mathrm{ml}$, with no Candida genus fungi found in 12 patients (40\%). In the two latter cases, no treatment was conducted.

No other opportunistic pathogenic microflora of the cervical canal was found.

In colposcopic examination, it was found that it is only in 5 cases (16.6\%) that cicatricial deformity was the monopathology while it was combined with the ectropion of the uterine cervix in the remaining 25 cases (83.3\%), and in 4 cases $(13.3 \%)$, the abnormal colposcopic condition (acetowhite epithelium, mosaic, punctation, iodine-negative areas) was found in the combination with the cervical ectropion.

According to the uterine cervix ultrasonography findings, in all patients, defects were identified at the places of ruptures, and endocervix cysts were found in 23 cases (76.6\%), which confirmed the presence of an inflammatory process.

Anti-inflammatory therapy was conducted for the inflammatory processes detected. When having verified the STIs, candidiasis, and bacterial vaginosis, local therapy was conducted which included antibacterial and antifungal agents, and the microbiocenosis was also corrected.

After the therapy conducted, the result of the treatment was assessed in 14 days. In 28 cases (93.3\%), the patients were completely cured, and in 2 cases $(6.6 \%)$ there was an inflammatory process but it was already not a pronounced leukocytosis but a moderate one.

The HCR HPV with the quantitative viral load of over $3 \mathrm{Lg}$ was detected in 4 patients (13.3\%), and in 26 patients (86.6\%), the HPV HCR was either not detected or its quantitative load was less than $3 \mathrm{Lg}$.

The content of immunoglobulines of various classes $(\lg A, \lg G, \lg M)$ in the venous blood serum and in the mucous secretion of the cervical canal of the patients examined before the treatment and 2 months after the surgical treatment in order to evaluate the local inflammation of the female lower reproductive tract led to conclusions that the level of immunoglobulines before the treatment was below the norm in all the patients (100\%), and in 2 months after the integrated treatment it was registered that 27 patients (90\%) had the level of immunoglobulines within the reference values.

After normalizing the vaginal biocenosis, the Superlimf 25 units immunomodulating agent in the form of vaginal suppositories once every $24 \mathrm{~h}$ for 10 days was prescribed to all patients included into the research, as another stage of the preoperative preparation. The agent was developed over 15 years ago at the immunology department of the Federal state budget-funded educational institution of higher education "N. I. Pirogov RSRMU". Superlimf 25 units is an immunomodulating agent having both antiviral and antimicrobial effect. The agent stimulates functional activity of the phagocytic type cells (monocytes and neutrophils): it activates phagocytosis and the production of cytokines (IL -1 , PNO). Under the effect of exogenic cytokines of Superlimf 25 units, the native cytokine background of tissues is altered, which induces the flow of mononuclear phagocytes into the affected area, due to which the inflammatory response gains a local and less pronounced nature. Superlimf 25 units boosts the immunity under infections and inflammatory diseases of the urogenital tract (20).

The surgical treatment was conducted at the first stage of the menstrual cycle after the preoperative preparation conducted. One day before the operative intervention and on the day of surgery, lavage of the vagina was performed by means of ultrasonic cavitation with the Fotek device. For the lavage, water solutions of chlorhexidine $0.05 \%$ and sodium chloride $0.9 \%$ were applied. $100-500 \mathrm{ml}$ of the solution was used at the 50 units ultrasound level and the maximum consumption of the liquid. The time of lavage was 2-5 minutes.

All patients were subdivided into 2 groups: the first group included 19 patients (63.3\%) who underwent plastic surgery of the uterine cervix by the dissection method. The scar tissue was excised within the healthy tissues, and the continuity of the uterine cervix was restored by individual vicryl sutures applied in two rows: the muscular-muscular ones and the muscular-mucous ones. The second group was composed of 11 patients (36.6\%) on whom conical cervical amputation was performed, with the anterior and posterior labia formed by individual vicryl Sturmdorf sutures and individual vicryl sutures applied on the sides.

The term of staying at the inpatient department amounted to 4-6 days or more. None of the patients has had any complications found.

On the $10^{\text {th }}$ day after the operative treatment, post-operative care using the Flamena liposomal gel coating with dihydroquercetin in order to stimulate regenerative processes, reduce the wound healing time, jugulate inflammatory processes of various etiology, and provide anti-edema action was prescribed for all patients. The liposomal complex contains dihydroquercetin - the natural antioxidant, lecithin - the complex of phospholipids, glycine - aminoacid, and sangviritrin - a natural origin agent. 
The Flamena liposomal antioxidant and phospholipid complex is presented in the form of applicators. All patients administered the gel at the dose of 1 applicator per night into the vagina for 10 days. No side effects or allergic reactions were found in any of the patients.

In order to evaluate the efficiency of the treatment, check-up examination was conducted in 2 and in 4 months after the operative treatment which included:

- detection of the content of immunoglobulines of various classes $(\lg A, \lg G, \lg M)$ in the venous blood serum and in the mucous secretion of the cervical canal in the patients examined in 2 months after the surgical treatment in order to identify the local immunity after the treatment conducted; as it has already been noted, the level of immunoglobulines was within the reference values in 27 patients (90\%).

- ultrasonography on day 5-7 of the menstrual cycle in 2 months after the treatment conducted; according to its results, it has been found that the uterine cervix was formed correctly in all patients, no ligatures were detected, and no endocervix cysts were found in any case.

- colposcopic examination in 2 and 4 months after the operative treatment. In all patients, the uterine cervix was formed correctly, ligatures were not detected, the external os was rounded or slit-like. In the extended colposcopic examination, 14 patients (46.6\%) had a satisfactory colposcopic condition (the junction of stratified squamous and cylindrical epithelia was visualized in the area of the external os, with the type 1 or 2 transformation area), and the colposcopic condition was unsatisfactory in 16 patients (53.3\%) (the junction of stratified squamous and cylindrical epithelia was not visualized, with the type 3 transformation area). None of the patients had an abnormal colposcopic condition.

In the histological examination, none of the patients had an acute cervicitis, which confirms the efficiency of antiinflammatory therapy in the preoperative preparation. In 18 patients (60\%), the chronic cervicitis was found according to the histological opinion, and CIN I was detected in one patient (3.3\%).

During examination of the uterine cervix in mirrors, it was registered that all patients had the complete epithelization, with a better progress of the reparative process, smaller edema, faster epithelization, and improved microcirculation noted.

So, the necessary constituent of post-operative care is acceleration of regeneration processes, improved microcirculation and the fastest epithelization of tissues stimulated by the Flamena liposomal antioxidant and phospholipid complex.

\section{CONCLUSIONS}

When diagnosing cicatricial deformities of the uterine cervix, it is important not only to detect gynecological criteria (localization of the ruptures, the presence of one or more ruptures, the presence of any ruptures reaching the vaginal vault or not) but also to conduct the integrated examination of the female patients (microscopic examination of the intensity of leukocytosis in the vaginal and cervical secretion; cytological examination; bacteriological inoculation from the cervical canal for detection of causative agents, sexually transmitted infections (STIs) by the PCR method, detection of the human papillomavirus (HPV), and the content of immunoglobulines of various classes $(\lg A, \lg G, \lg M)$ in the venous blood serum and in the mucous secretion of the cervical canal; colposcopic examination; ultrasonography of the uterine cervix and histological examination).

The data obtained allow arguing positively that it is important to conduct the preoperative diagnostics and treatment of patients having cicatricial deformity of the uterine cervix using the anti-inflammatory, antibacterial and antiviral agents, as well as the Superlimf 25 units immunomodulating agent in order to improve the local immunity and create a favorable background for surgical treatment.

Assessment of the efficiency of the Flamena liposomal gel coating with dihydroquercetin used intravaginally in patients having cicatricial deformity of the uterine cervix after surgical treatment has shown that this agent accelerates regenerative processes of the wound surface of tissues and contributes to the full-fledged epithelization of the uterine cervix in the post-operative period, which allows recommending this agent for clinical practice.

The algorithm suggested by the authors and including the preoperative preparation, surgical treatment and postoperative care can be used as an integrated approach to the management of patients having cicatricial deformity of the uterine cervix. 


\section{REFERENCES}

1. Krasnopolskiy VI. Obligatory forms of precancer and invasive cervical cancer: a manual for doctors edited by the academician of the RAS. Moscow: SIMK; 2017.

2. Dubrovina SO, Ardintseva OA, Krasilnikova LV. On diagnostics, prevention and therapy of the uterine cervix pathology. Gynecology, 2018;20(5):33-6. https://doi.org/10.26442/2079-5696_2018.5.33-36

3. Bruni L, Barrionuevo-Rosas L, Albero G. WHO/ICO Information Centre on HPV and Cancer. Russian Federation: Human Papillomavirus and Related Cancers, Fact Sheet; 2017.

4. National Cancer Institute $(\mathrm{NCl})$. Cancer Stat Facts: cervical cancer. $\mathrm{NCl}$ website. Available at: https://seer.cancer.gov/statfacts/html/cervix.html (Accessed June 28, 2018).

5. Melnikow J, Henderson JT, Burda BU. Screening for Cervical Cancer With High-Risk Human Papillomavirus Testing: Updated Evidence Report and Systematic Review for the US Preventive Services Task Force. JAMA 2018;320(7):687-705. https://doi.org/10.1001/jama.2018.10400 PMid:30140883

6. Mezhevitinova EA, Donnikov AE, Prilepskaya VN. Cervical dysplasia. The role of cytokine therapy in enhancing the efficiency of treatment. Obstetrics and gynecology: news, opinions, training, 2019;7(4):66-71.

7. Prilepskaya VN. Uterine cervix cancer prevention. Moscow: Medpress-inform; 2006.

8. Bairamova GR, Baranov II, Arslanyan KN, Amirkhanyan AS, Chernova VF. Diseases of the lower genitalia associated with the papilloma viral infection: radical treatment or immunotherapy? Obstetrics and gynecology: news, opinions, training. Analytical Reviews, 2017;4(18):13-9.

9. Wheeler CM, Hunt W.C., Schiffman M., Castle P.E. Human papillomavirus genotypes and the cumulative 2-years risk of cervical precancer. J. Infect. Dis, 2006;194:1291-9. https://doi.org/10.1086/507909 PMid:17041856

10. Novikov Al, Kononov AV, Vaganova IG. Sexually transmitted infections and exocervix. Moscow: Medicina, 2010.

11. Sergheeva YuA, Gustovarova TA, Kuzminykh VV. Cicatricial deformity of the uterine cervix: risk factors, remote results of plastic surgery in the early postpartum period. Smolensk: Smolensk medical almanac; 2017.

12. Karakhalis LYu, Petrenko VS, Petrenko SI. The informative value of colposcopic examination in inflammatory diseases of the uterine cervix. Obstetrics and gynecology: news, opinions, training, 2019;7(4):20-4.

13. Marchetta J. Descamps P. Colposcopy. Techniques and diagnostics: a practical manual. Moscow: MedpressInform, 2014.

14. Girardi F, Reich O, Tamussino C, Pickel H. Burghardt's colposcopy and cervical pathology. Moscow: MedpressInform, 2018.

15. Rogovskaya SI. Practical colposcopy. Moscow: GEOTAR-Media, 2018.

16. Prilepskaya VN. Diseases of the uterine cervix and genital infections. Moscow: GEOTAR-Media, 2016.

17. Abakarova PR, Prilepskaya VN, Mezhevitinova EA, Donnikov AE. Local cytokine therapy in the integrated treatment of HPV-associated diseases of the uterine cervix. Gynecology, 2019;21(1):28-32. https://doi.org/10.26442/20795696.2019.1.190284

18. Seresini S, Origoni M, Lillo F. IFN-gamma produced by human papillomavirus-18 E6-spesific CD4+ T-cells predicts the clinical outcome after surgery with high-grade cervical lesions. J Immunol, 2007;179:76-83. https://doi.org/10.4049/jimmunol.179.10.7176 PMid:17982110

19. Chernova NI, Perlamutrov YuN, Petrova IS. The efficiency of the antimicrobial peptides complex in treatment of relapsing inflammatory diseases of the lower urogenital tract conditioned by a combined infection. Clinical dermatology and venereology, 2018;17(3):70-9. https://doi.org/10.17116/klinderma201817380

$\diamond \diamond \diamond \diamond \diamond \diamond \diamond$

http://www.ejgm.co.uk 